\title{
Prevalence of antimicrobial resistant pathogens from blood cultures: results from a laboratory based nationwide surveillance in Ghana
}

\author{
Japheth Awuletey Opintan * ${ }^{*}$ and Mercy Jemima Newman
}

\begin{abstract}
Background: Blood stream infections (BSI) are critical medical conditions with high morbidity and mortality. There is paucity of information on BSI from surveillance studies in Ghana.

Aim: This study sought to demonstrate how useful BSI data can be gleaned from population-based surveillance, especially from resource-limited settings.

Methods: Data from a nationwide surveillance of antimicrobial drug resistance (AMR) in Ghana were extracted and analyzed. Secondly, we revived archived Staphylococcus aureus isolates from blood cultures that were cefoxitin resistant (CRSA), and screened these for protein A (spa) and mec A genes.

Results: Overall blood culture positivity was 11.2\% (714/6351). All together, participating laboratories submitted 100 multidrug resistant blood culture isolates (Gram-negative $=49$ and Gram-positive $=51$ ). Prevalence of some Gram-negative isolates was as follows; Escherichia coli (20.4\%), Pseudomonas aeruginosa (16.3\%), Enterobacter spp. (14.3\%), Salmonella serotype Typhi (8.2\%) and Non-typhoidal Salmonella [NTS] (8.2\%). Gram-positive pathogens included Staphylococcus aureus (66.7\%), coagulase negative S. aureus [CoNS] (17.6\%) and Streptococcus pneumoniae (11.8\%). No methicillin resistance was confirmed in our CRSA isolates. Most blood stream associated infections were from inpatients (75\%) and cultured bacteria were resistant to common and cheaper antimicrobials.
\end{abstract}

Conclusion: E. coli and S. aureus are common pathogens associated with BSI in Ghana and they are resistant to several antimicrobials. Active and continuous AMR surveillance can serve multiple purposes, including data generation for BSI.

Keywords: Bloodstream infections, Antimicrobial drug resistance, Blood culture, Surveillance, Methicillin resistance

\section{Background}

Bloodstream infections (BSI) are important cause of morbidity and mortality, that require prompt and appropriate empiric therapy before blood culture results are ready. The management of BSI is further complicated in an era of increasing antimicrobial resistance (AMR) [1]. Empiric antimicrobial therapy when administered early in the course of BSI has the potential to save lives [2]. However, the epidemiology of the causative agents are not static, constantly changing over time [3]. Especially

\footnotetext{
* Correspondence: jaopintan@ug.edu.gh

Medical Microbiology Department, School of Biomedical and Allied Health Sciences, Korle-Bu, P. O. Box KB 4236, Accra, Ghana
}

in resource limiting settings with inadequate laboratory support for culture and antimicrobial surveillance, BSI management is further complicated.

Several resource-rich countries have surveillance systems in place to monitor changing trends of infections including BSI. Examples include the Health Protection Agency (HPA) in England [3] and the Canadian Antimicrobial Resistance Alliance (CARA) [4]. These surveillance systems readily provide useful data on microorganisms of clinical significance, including those associated with BSI, providing clues on aetiological trends and AMR. Active surveillance systems are non-existent in most resource-limited countries including Ghana, in spite of the high disease in 
these countries. Several local studies, especially in tertiary care facilities have been done in Ghana and elsewhere, but these are either disease condition or age specific [5-8]. In a previous study, we presented the overall results of a nationwide surveillance. In that study, only about $40 \%$ of participating hospitals provided data on blood culture results. In the present study, we focused on blood stream infections and analyzed the data on blood culture results that we did receive by those $40 \%$ of participating hospitals. We did this to emphasize the importance of more detailed information that can be obtained from more detailed reporting, and to encourage better participation in such nationwide surveillance.

\section{Methods}

Data on bloodstream infections (BSI), including patient information, bacterial isolates and their antibiogram were electronically extracted from our surveillance records [9]. The Department of Medical Microbiology, School of Biomedical and Allied Health Sciences (DMSBAHS) coordinated this surveillance, June-November 2014. Participating laboratories used in-house standard operating procedures to culture and to identify bacteria from all specimens submitted for bacteriological analysis. Antimicrobial susceptibility testing was done by the disc diffusion test, in accordance with the Clinical and Laboratory Standards Institute [10] guidelines. Multiple drug resistant isolates, defined as resistance to more than two antimicrobial drug classes were submitted to DM-SBAHS. The location and description of participating laboratories are described elsewhere [9] and included Teaching, Regional, District and Faithbased hospitals in Ghana.

We analyzed data on all blood cultures submitted by participating laboratories to DM-SBAHS. To confirm methicillin resistant Staphylococcus aureus (MRSA), all stored cefoxitin-resistant $S$. aureus (CRSA) isolates were revived on blood agar plates. The identities of these CRSA were reconfirmed by Gram stain, catalase and tube coagulase tests. Genomic DNAs were extracted from pure CRSA isolates and were then screened by polymerase chain reaction for Staphylococcus-specific genes (spa and $m e c A$ ), using previous protocols [11]. Data on BSI were stratified by age, isolate source and hospital facility and were summarized in tables or percentages.

\section{Results}

In total, 11 of 18 (61.1\%) laboratories processed and submitted surveillance data on blood cultures. The overall culture positivity rate was $11.2 \%(714 / 6351)$ with great variabilities ranging between 4.7-27.3\% (Table 1). Total number of non-duplicate multidrug resistant blood isolates received from participating laboratories was 100 (Gram-negative, $n=49$; Gram-positive, $n=51$ ). Blood
Table 1 Blood cultures processed and data submitted during surveillance

\begin{tabular}{llll}
\hline Laboratory code & $\begin{array}{l}\text { Total blood cultures } \\
\text { processed }\end{array}$ & $\begin{array}{l}\text { Positives, } \\
\text { rates (\%) }\end{array}$ & $\begin{array}{l}\text { Multi-drug resistant } \\
\text { isolates submitted } \\
\text { during surveillance }\end{array}$ \\
\hline BaS & 315 & $86(27.3)$ & 41 \\
$\mathrm{AcL}$ & 226 & $54(23.9)$ & 16 \\
$\mathrm{WsE}$ & 505 & $43(8.5)$ & 14 \\
$\mathrm{NoT}$ & 1376 & $102(7.4)$ & 13 \\
$\mathrm{AsK}$ & 3610 & $398(11.0)$ & 7 \\
$\mathrm{CeU}$ & 13 & $3(23.1)$ & 1 \\
$\mathrm{AsO}$ & 146 & $16(10.9)$ & 1 \\
$\mathrm{BaB}$ & 21 & $1(4.7)$ & 1 \\
EaK & 95 & $8(8.4)$ & 1 \\
UeB & 44 & $3(6.8)$ & 1 \\
Total & 6351 & 714 & 100 \\
\hline
\end{tabular}

Teaching hospitals: Tamale (NoT) and Komfo Anokye (AsK). Regional hospitals: Sunyani (Bas), Eastern (EaK) and Upper East (UeB). District hospital: LEKMA (AcL), Faith base: St Patrick, Offinso (AsO), St Patrick Holy Family, Berekum (BaB). Quasi-government hospital: University, Cape Coast (CeU). Zonal public health reference laboratory: Sekondi (WsE)

culture Gram-negative (GN) isolates included Escherichia coli (20.4\%), Pseudomonas aeruginosa (16.3\%), Enterobacter spp. (14.3\%), Salmonella Typhi (8.2\%) and Non-Typhoidal Salmonella [NTS] (8.2\%). E. coli was predominantly isolated from patients aged older than 10 years. Other GN rods like typhoidal and NTS were mostly recovered from children less than 10 years of age [Table 2]. Gram-positive (GP) isolates included Staphylococcus aureus (66.7\%), coagulase negative Staphylococcus [CNS] (17.6\%) and Streptococcus pneumoniae (11.8\%). Staphylococus and Streptococcus were generally recovered from children less than 10 years. However many patients with staphylococcal associated BSI had missing data for age (Table 2). Multidrug resistant isolates were predominately recovered from inpatients $(>70 \%)$. Tables 3 and 4 show the prevalence of antimicrobial drug resistance of epidemiologically important GN and GP bacterial isolates, respectively.

In total, 4 of 6 CRSA were successfully revived and molecularly screened for MRSA (mecA and spa genes). None of these tested positive for MRSA, both negative for $m e c A$ and spa genes.

\section{Discussion}

To inform empiric antibiotic guidelines at institutional level, one requires regular review of pathogen spectrum and antibiogram. Critical medical information to manage BSI could be gleaned from population-based or nosocomial surveillance systems $[6,8,12-16]$. However, the current trend for investigating BSI is to use populationbased approaches $[15,17]$. Information from such systems becomes even more useful when they are designed 
Table 2 Gram negative and positive multiple drug resistant isolates from surveillance records

\begin{tabular}{|c|c|c|c|c|c|c|c|c|c|c|}
\hline \multirow[t]{2}{*}{ Bloodstream isolates } & \multirow[t]{2}{*}{ n (\%) } & \multicolumn{3}{|c|}{ Isolate source } & \multicolumn{6}{|c|}{ Age categories/years } \\
\hline & & In-patient & Out-patient & Not indicated $^{a}$ & $\overline{<1}$ & $1-10$ & $11-30$ & $31-50$ & $>50$ & Not indicated $^{a}$ \\
\hline \multicolumn{11}{|l|}{ Gram negatives $(n=49)$} \\
\hline Escherichia coli & 10 & 9 & 1 & - & 1 & - & 3 & 2 & 2 & 2 \\
\hline Salmonella Typhi & 4 & 2 & 2 & - & - & 4 & - & - & - & - \\
\hline Non-Typhoidal Salmonella & 4 & 4 & - & - & 2 & 2 & - & - & - & - \\
\hline Enterobacter spp & 7 & 6 & 1 & - & 3 & - & 1 & 1 & - & 2 \\
\hline Serratia marcescens & 4 & 4 & - & - & - & 2 & - & - & 1 & 1 \\
\hline Serratia spp & 2 & 2 & - & - & - & 2 & - & - & 1 & 1 \\
\hline Klebsiella pneumoniae & 2 & 2 & - & - & - & 2 & - & - & - & - \\
\hline Citrobacter korseri & 1 & - & 1 & - & 1 & - & - & - & - & - \\
\hline Citrobacter spp & 5 & 5 & - & - & 2 & 3 & - & - & - & - \\
\hline Acinetobacter & 1 & 1 & - & - & 1 & - & - & - & - & - \\
\hline Pseudomonas aeruginosa & 8 & 6 & 1 & 1 & 3 & 1 & - & 1 & 2 & 1 \\
\hline Proteus mirabilis & 1 & 1 & - & - & - & - & 1 & - & - & - \\
\hline \multicolumn{11}{|l|}{ Gram positives $(n=51)$} \\
\hline Staphylococcus aureus & 34 & 23 & 8 & 3 & 11 & 10 & 3 & 1 & 2 & 7 \\
\hline CNS & 9 & 8 & - & 1 & 3 & 2 & 1 & - & 1 & 2 \\
\hline Streptococcus pneumoniae & 6 & 5 & 1 & - & 2 & 3 & - & - & - & 1 \\
\hline Streptococcus pyogenes & 1 & 1 & - & - & 1 & - & - & - & - & - \\
\hline Enterococcus spp & 1 & - & - & - & - & - & 1 & - & - & - \\
\hline Totals & 100 & 75 & 15 & 5 & 30 & 29 & 10 & 5 & 8 & 16 \\
\hline
\end{tabular}

CNS, Coagulase negative Staphylococcu

-indicates not applicable

anavailable date

to capture additional indicators. For example, illness severity as indicated by Pitt bacteraemia score, [18] receipt of adequate antimicrobial regimen, [19] primary source of infection, and the presence or absence of malignancies [20]. Previous studies on BSI in Ghana were limited to tertiary facilities and restricted to particular age category $[8,16,21,22]$. The current study used populationbased surveillance to investigate BSI.

Overall blood culture positivity rate was only about $11 \%$ with a wide variability range (between $4.7 \%$ to $27 \%$ )

Table 3 Antibiogram of epidemiologically important Gram negative blood culture isolates

\begin{tabular}{|c|c|c|c|c|c|}
\hline Antimicrobial agent & $\begin{array}{l}\text { E. coli }(n=10) \\
n,(\% \text { resistant })\end{array}$ & $\begin{array}{l}\text { S. Typhi }(n=4) \\
\text { n, (\% resistant) }\end{array}$ & $\begin{array}{l}\text { NTS }(n=4) \\
\text { n, (\% resistant) }\end{array}$ & $\begin{array}{l}\text { Pseudomonas }(n=8) \\
\mathrm{n},(\% \text { resistant })\end{array}$ & $\begin{array}{l}\text { Enterobacter }(n=7) \\
n,(\% \text { resistant })\end{array}$ \\
\hline Ampicillin & $10(100)$ & $4(100)$ & $3(100)$ & $8(62.5)$ & $7(100)$ \\
\hline Cefuroxime & $8(87.5)$ & $3(66.6)$ & $3(50)$ & $6(66.7)$ & $7(85.7)$ \\
\hline Cefotaxime & $9(88.9)$ & $4(50)$ & $3(60)$ & $8(87.5)$ & $7(85.7)$ \\
\hline Meropenem & $8(12.5)$ & $3(0)$ & $4(0)$ & $5(20)$ & $5(0)$ \\
\hline Amikacin & $8(0)$ & $2(0)$ & $2(0)$ & $8(12.5)$ & $3(33.3)$ \\
\hline Gentamicin & $10(30)$ & $4(25)$ & $3(50)$ & $8(25)$ & $5(80)$ \\
\hline Ciprofloxacin & $10(60)$ & $2(100)$ & ND & $8(12.5)$ & $4(25)$ \\
\hline Levofloxacin & $7(42.9)$ & $2(50)$ & ND & ND & ND \\
\hline Trimethoprim/Sulfamethoxazole & $10(100)$ & $4(50)$ & $3(100)$ & $8(75)$ & $7(71.4)$ \\
\hline Chloramphenicol & $9(100)$ & $4(50)$ & $3(100)$ & $7(71.4)$ & $7(57.2)$ \\
\hline Tetracycline & $10(100)$ & $4(50)$ & $3(50)$ & $8(62.5)$ & $5(80)$ \\
\hline
\end{tabular}


Table 4 Antibiogram of epidemiologically Gram positive blood culture isolates

\begin{tabular}{llll}
\hline Antimicrobial agent & Staph aureus $(n=34)$ & CNS $(n=9)$ & $\begin{array}{l}\text { S. pneumoniae }(n=6) \\
n, \% \text { resistance }\end{array}$ \\
\hline Penicillin & $n, \%$ resistance & $n, \%$ resistance & $6(0)$ \\
Ampicillin & $26(23.1)$ & $6(0)$ & $6(0)$ \\
Cloxacillin & $30(100)$ & $8(100)$ & ND \\
Flucloxacillin & $14(100)$ & $4(0)$ & ND \\
Augmentin & $17(14.3)$ & $1(0)$ & ND \\
Cefuroxime & $12(75)$ & ND & ND \\
Cefotaxime & $26(53.8)$ & $6(16.7)$ & ND \\
Gentamicin & $14(71.4)$ & $8(62.5)$ & $6(16.7)$ \\
Ciprofloxacin & $30(30)$ & $8(25)$ & $6(50)$ \\
Trimethoprim/Sulfamethoxazole & $29(34.5)$ & $8(62.5)$ & $6(0)$ \\
Erythromycin & $30(70)$ & $8(100)$ & $6(66.7)$ \\
Tetracycline & $5(64)$ & $8(100)$ & $6(83.3)$ \\
Vancomycin & $31(77.5)$ & ND & $2(0)$
\end{tabular}

CNS Coagulase negative Staphylococcus, ND indicates not done

across the 10 surveillance sites in Ghana. These rates have serious implications on patient care and also reflect blood culture capabilities in the respective hospitals. A combination of the following reasons may apply; some patients already on antibiotics prior to specimen collection, misdiagnosis from clinicians or laboratories using inadequate culture methods. It is worth noting that most of the regional hospitals in this study use the BACTEC blood cultures systems, but are usually short of one supply or the other. Alizadeh and colleagues however showed that both BACTEC and conventional blood culture methods have high validity [23]. We propose that the BACTEC and conventional blood culture methods should be used complementarily, especially in resourcelimited countries, where consumables for the BACTEC are often in short supply. Infrastructure for blood culture services, whether BACTEC or conventional should be available for BSI diagnosis and management. Several studies in Ghana [8, 22] had blood culture positivity rates greater than $20 \%$ and in a large Indian study with over 135,000 cultures, a 14\% rate was observed [24]. In a meta-analysis review, Reddy and colleagues identified bloodstream infections in $13.5 \%$ and $8.2 \%$ adults and children, respectively, with overall in-hospital case fatality rate of about 18\% [25]. Our analysis did not follow up on patient outcomes but poor laboratory infrastructure and inadequate diagnostics, often results in poor treatment outcomes, sometimes confusing malaria bloodstream infections with other pathogens [25].

We found slightly more Gram-positive bacteria (GPB) in association with BSI in the current study, contrary to the Gram-negative bacteria (GNB) observed in several studies in Ghana $[8,22]$ and elsewhere [26]. Among the
GNB, E coli was responsible for about a fifth of the BSI in the current study. Elsewhere, patient specific studies have generally associated $E$. coli to BSI $[6,8,22]$. On the contrary, in Europe, though $E$ coli was previously topping the list of organisms associated with BSI, this has currently given way to MRSA [3, 14]. E coli associated BSI in most cases is secondary to focal infections such as urinary tract, abdominal, hepato-biliary sepsis, or surgical site infections [3]. An association with central vascular lines has also been reported [13]. Besides E. coli, S. Typhi and NTS were other GNB with higher prevalence, especially among children with ages less than 10 years. Compared to resource-rich countries where Salmonella (whether typhoidal or nontyphoidal) is almost never implicated in BSI $[3,4,14]$, persistently, this organism is a common cause of $\mathrm{GN}$ sepsis in resource limiting countries $[7,8,22,24,27]$. It is generally difficult to distinguish between community and hospital acquired BSI. However, Pseudomonas is often associated with hospital-acquired BSI, often related to indwelling devices. In the current study Pseudomonas was responsible for more than $16 \%$ of all GN BSI.

Among the GPB, Staphylococcus and CNS were the predominant organisms, together accounting for greater than $80 \%$ of all GP sepsis. Staphylococcus was found more in patients less than 30 years of age, whilst CNS was found in children less than 10 years. Higher frequencies of GPB have been implicated in some earlier BSI studies from the USA and Canada [14]. GPB dominates in places where more prosthetic devices, mainly intravenous catheters, invasive procedures and specific procedures are done [28]. We did not confirmed MRSA in the current study. Meanwhile, studies from the largest 
tertiary hospital in Ghana have reported MRSA, primarily based on cefoxitin resistance $[8,22]$. This tertiary hospital did not submit data for the current surveillance. MRSA associated BSI has been reported elsewhere. In England, mandatory and voluntary surveillance systems helped in reducing high MRSA bacteraemia [29]. Streptococcus pneumoniae, the next frequent GPB were predominantly found in children less than 5 years of age, comparable to other observations [8].

The high prevalence of antimicrobial drug resistance (AMR) observed in the GNB associated with BSI is alarming. Among the GNB, it appears meropenem and amikacin are the last resort antimicrobials. The hospitals in our surveillance reported and submitted data only on multi-drug resistant isolates, and we did not have information on the isolates susceptible. However, management of BSI in these patients with these high resistant bacteria will be difficult and may have bad outcomes. Extended spectrum beta-lactamase (ESBLs) and carbapenemase producing enterobacteriaceae (CRE) isolates in the current analysis is a worrying concern. These hydrolyzing enzymes render these antimicrobials ineffective, but are sparingly screened for in Ghana, especially, in bloodstream infections $[8,30]$. Elsewhere, relatively high levels of these enzymes have been found in bloodstream isolates [31]. Scaling up of laboratory capacity in resourcelimited countries like Ghana is urgently needed. In the absence of molecular assays for these enzymes, phenotypic assays would be helpful for patient management. GPB like Staphylococcus and CNS were also resistant to older antibiotics. We did not observe any MRSA (by molecular assay) and vancomycin resistant Staphylococcus in the current study, contrarily to other investigations in Ghana [8]. However, it is important to highlight that all Staphylococcus aureus isolates submitted by participating laboratories were resistant to cloxacillin (Table 4). Since cefoxitin is a better inducer for mecA (MRSA), it is currently preferred to oxacillin. A polymerase chain reaction (PCR), targeting the mecA gene remains the gold standard for MRSA confirmation [32]. However, PCR is seldom used in routine clinical laboratories because of logistics and inability to standardized laboratory protocols. The current study is not without limitations, which includes incomplete data sets and detailed clinical information. No clinical information was available about the patients from the participating laboratories. It is therefore difficult to differentiate between community acquired and hospital acquired BSIs [15, 17]. Complete clinical information, if available will help in ruling out some possible skin contaminants, which many include some CNS. Information on blood culture contaminants has implication on cost and practice. Finally, the relatively fewer numbers of bacterial isolates tested in any particular species limits the power of our study.

\section{Conclusion}

E. coli and Salmonella (typhoidal and NTS), Staphylococcus aureus, CNS and Streptococcus pneumoniae are often associated with BSI in Ghana. Most of these organisms are resistant to common antibiotics. Active and continuous surveillance systems will play a dual role in monitoring BSI and AMR.

\section{Acknowledgement \\ We acknowledge all the laboratories that participated in this study. Reuben Arhin Essel, Amos Akanwena, Mary Osei and Abena Lawson are appreciated for providing technical assistance. Beverly Egyir performed MRSA screening test and we are grateful to her.}

\section{Funding}

None to declare.

\section{Availability of data and materials}

Data and materials have been provided in the main manuscript. Additional information can be found in [9].

Authors' contributions

JAO and MJN designed the study. JAO analyzed data and drafted manuscript. Both authors read and approved the final manuscript.

\section{Competing interests}

The authors declare that they have no competing interests.

\section{Consent for publication}

Not applicable.

\section{Ethics approval and consent to participate}

Not applicable in this study as analysis was done on previous surveillance data. Ethical issues were considered under previous study.

\section{Publisher's Note}

Springer Nature remains neutral with regard to jurisdictional claims in published maps and institutional affiliations.

Received: 15 January 2017 Accepted: 9 June 2017

Published online: 13 June 2017

\section{References}

1. Fabbro-Peray P, Sotto A, Defez C, Cazaban M, Molinari L, Pinède M, et al. Mortality attributable to nosocomial infection: a cohort of patients with and without nosocomial infection in a French university hospital. Infect Control Hosp Epidemiol. 2007;28:265-72.

2. Munson EL, Diekema DJ, Beekmann SE, Chapin KC, Doern GV. Detection and treatment of bloodstream infection: laboratory reporting and antimicrobial management. J Clin Microbiol. 2003:41:495-7.

3. Wilson J, Elgohari S, Livermore DM, Cookson B, Johnson A, Lamagni T, et al. Trends among pathogens reported as causing bacteraemia in England, 2004-2008. Clin Microbiol Infect. 2011;17:451-8.

4. Adam HJ, DeCorby M, Rennie R, Karlowsky JA, Hoban DJ, Zhanel GG, et al. Prevalence of antimicrobial resistant pathogens from blood cultures from Canadian hospitals: results of the CANWARD 2007-2009 study. Diagn Microbiol Infect Dis. 2011:69:307-13.

5. Hamer DH, Darmstadt GL, Carlin JB, Zaidi AK, Yeboah-Antwi K, Saha SK, et al. Etiology of bacteremia in young infants in six countries. Pediatr Infect Dis J. 2015;34:e1-8

6. Krakash KP, Vinod A, Geethanjali PP. Bloodstream bacterial pathogens and their antibiotic resistance pattern in Dhahira Region, Oman. Oman Med J. 2011;26:240-79.

7. Nielsen MV, Sarpong N, Krumkamp R, Dekker D, Loag W, Amemasor S, et al. Incidence and characteristics of bacteremia among children in rural Ghana. PLoS One. 2012;7:e44063.

8. Obeng-Nkrumah N, Labi AK, Addison NO, Labi JE, Awuah-Mensah G. Trends in paediatric and adult bloodstream infections at a Ghanaian referral hospital: a retrospective study. Ann Clin Microbiol Antimicrob. 2016;15:49. 
9. Opintan JA, Newman MJ, Arhin RE, Donkor ES, Gyansa-Lutterodt M, MillsPappoe W. Laboratory-based nationwide surveillance of antimicrobial resistance in Ghana. Infect Drug Resist. 2015;8:379-89.

10. CLSI. Clinical Laboratory Standards Institute: Performance standards for antimicrobial susceptibility testing: twenty-second informational supplement;[. provides updated tables for. M02-A11 and M07 .... (2012).

11. Egyir B, Guardabassi L, Sørum M, Nielsen SS, Kolekang A, Frimpong E, et al. Molecular epidemiology and antimicrobial susceptibility of clinical Staphylococcus aureus from healthcare institutions in Ghana. PLoS One. 2014;9:e89716.

12. Al-Hasan MN, Lahr BD, Eckel-Passow JE, Baddour LM. Seasonal variation in Escherichia coli bloodstream infection: a population-based study. Clin Microbiol Infect. 2009;15:947-50.

13. Albrecht SJ, Fishman NO, Kitchen J, Nachamkin I, Bilker WB, Hoegg C, et al. Reemergence of gram-negative health care-associated bloodstream infections. Arch Intern Med. 2006;166:1289-94.

14. Biedenbach DJ, Moet GJ, Jones RN. Occurrence and antimicrobial resistance pattern comparisons among bloodstream infection isolates from the SENTRY Antimicrobial Surveillance Program (1997-2002). Diagn Microbio Infect Dis. 2004:50:59-69.

15. Goto M, Al-Hasan MN. Overall burden of bloodstream infection and nosocomial bloodstream infection in North America and Europe. Clin Microbiol Infect. 2013;19:501-9.

16. Gross U, Amuzu SK, de Ciman R, Kassimova I, Gross L, Rabsch W, et al. Bacteremia and antimicrobial drug resistance over time, Ghana. Emerg Infect Dis. 2011;17:1879-82.

17. Laupland KB, Church DL. Population-based epidemiology and microbiology of community-onset bloodstream infections. Clin Microbiol Rev. 2014;27: 647-64.

18. Paterson DL, Ko WC, Von Gottberg A, Mohapatra S, Casellas JM, Goossens H, et al. International prospective study of Klebsiella pneumoniae bacteremia: implications of extended-spectrum beta-lactamase production in nosocomial Infections. Ann Intern Med. 2004;140:26-32.

19. Kang $\mathrm{Cl}$, Kim SH, Park WB, Lee KD, Kim HB, Kim EC, et al. Bloodstream infections caused by antibiotic-resistant gram-negative bacilli: risk factors for mortality and impact of inappropriate initial antimicrobial therapy on outcome. Antimicrob Agents Chemother. 2005;49:760-6.

20. Al-Hasan MN, Lahr BD, Eckel-Passow JE, Baddour LM. Predictive scoring model of mortality in Gram-negative bloodstream infection. Clin Microbiol Infect. 2013;19:948-54

21. Acquah SE, Quaye L, Sagoe K, Ziem JB, Bromberger PI, Amponsem AA. Susceptibility of bacterial etiological agents to commonly-used antimicrobial agents in children with sepsis at the Tamale Teaching Hospital. BMC Infect Dis. 2013;13:89.

22. Labi AK, Obeng-Nkrumah N, Bjerrum S, Enweronu-Laryea C, Newman MJ. Neonatal bloodstream infections in a Ghanaian Tertiary Hospital: are the current antibiotic recommendations adequate. BMC Infect Dis. 2016;16:598.

23. Alizadeh AM, Kabiri Movahed R, Mohammadnia M. Comparative evaluation of conventional and BACTEC methods for detection of bacterial infection. Tanaffos. 2016;15:112-6.

24. Gandra S, Mojica N, Klein EY, Ashok A, Nerurkar V, Kumari M, et al. Trends in antibiotic resistance among major bacterial pathogens isolated from blood cultures tested at a large private laboratory network in India, 2008-2014. Int J Infect Dis. 2016;50:75-82.

25. Reddy EA, Shaw AV, Crump JA. Community-acquired bloodstream infections in Africa: a systematic review and meta-analysis. Lancet Infect Dis. 2010;10: 417-32.

26. Luzzaro F, Ortisi G, Larosa M, Drago M, Brigante G, Gesu G. Prevalence and epidemiology of microbial pathogens causing bloodstream infections: results of the OASIS multicenter study. Diagn Microbiol Infect Dis. 2011;69: 363-9.

27. Negussie A, Mulugeta G, Bedru A, All I, Shimeles D, et al. Bacteriological Profile and Antimicrobial Susceptibility Pattern of Blood Culture Isolates among Septicemia Suspected Children in Selected Hospitals Addis Ababa, Ethiopia. Int J Biol Med Res. 2015;6:4709-17.

28. Muñoz P, Cruz AF, Rodríguez-Créixems M, Bouza E. Gram-negative bloodstream infections. Int J Antimicrob Agents. 2008;32(Suppl 1):S10-4.

29. Pearson A, Chronias A, Murray M. Voluntary and mandatory surveillance for methicillin-resistant Staphylococcus aureus (MRSA) and methicillinsusceptible S. aureus (MSSA) bacteraemia in England. J Antimicrob Chemother. 2009;64(Suppl 1):i11-7.
30. Obeng-Nkrumah N, Twum-Danso K, Krogfelt KA, Newman MJ. High levels of extended-spectrum beta-lactamases in a major teaching hospital in Ghana: the need for regular monitoring and evaluation of antibiotic resistance. Am J Trop Med Hyg. 2013;89:960-4.

31. Myat TO, Hannaway RF, Zin KN, Htike WW, Win KK, et al. ESBL- and carbapenemase-producing enterobacteriaceae in patients with bacteremia, Yangon, Myanmar, 2014. Emerg Infect Dis. 2017;23:857-9.

32. Chomvarin C, Siripornmongcolchai T, Chaicumpar K, Limpaiboon T, Wongkham $\mathrm{C}$, et al. Evaluation of polymerase chain reaction, conventional and MRSA screen latex agglutination methods for detection of methicillinresistant, -borderline and -susceptible Staphylococcus aureus. Southeast Asian J Trop Med Public Health. 2004;35:879-85.

\section{Submit your next manuscript to BioMed Central and we will help you at every step:}

- We accept pre-submission inquiries

- Our selector tool helps you to find the most relevant journal

- We provide round the clock customer support

- Convenient online submission

- Thorough peer review

- Inclusion in PubMed and all major indexing services

- Maximum visibility for your research

Submit your manuscript at www.biomedcentral.com/submit
) Biomed Central 